\title{
Identification of Matrix Metalloproteinase 11 as a Prognostic Biomarker in Pancreatic Cancer
}

\author{
JUNGWHOI LEE ${ }^{1 *}$, JUNGSUL LEE $^{2 *}$ and JAE HOON KIM ${ }^{1,3}$ \\ ${ }^{1}$ Department of Applied Life Science, SARI, Jeju National University, Jeju, Republic of Korea; \\ ${ }^{2}$ Department of Bio and Brain Engineering, KAIST, Daejeon, Republic of Korea; \\ ${ }^{3}$ Subtropical/tropical Organism Gene Bank, Jeju National University, Jeju, Republic of Korea
}

\begin{abstract}
Background/Aim: The aim of this study was to investigate matrix metalloproteinase 11 (MMP11) as a promising biomarker in human pancreatic cancer. Materials and Methods: A consecutive eliminating method was used to select biomarker candidates in pancreatic cancer. $m R N A$ and protein expression levels of candidates were determined in tissues and whole blood samples of healthy donors and pancreatic cancer patients. The prognostic value of MMP11 was determined using various data-sets and Liptak's $Z$ analysis. Results: Analysis using Gene Expression Omnibus (GEO) database showed significantly higher MMP11 mRNA expression in pancreatic cancer tissues compared to that in various normal tissues. MMP11 protein was specifically expressed in pancreatic cancer tissues, but not in various normal or other cancer tissues. Secreted MMP11 levels could be measured using easily accessible techniques and whole blood samples of pancreatic cancer. In addition, high levels of MMPII were associated with poor prognosis of pancreatic cancer patients. Conclusion: MMP11 may be a promising prognostic biomarker for pancreatic cancer patients.
\end{abstract}

Pancreatic ductal adenocarcinoma, also known as pancreatic cancer, is one of the most aggressive diseases in the world. For patients with such cancer, the 5-year survival rate is below 7\% with a median survival of approximately 6 months

\footnotetext{
*These Authors contributed equally to this work.

Correspondence to: Lee Jungwhoi and Kim Jae Hoon, Department of Biotechnology, College of Applied Life Science, Jeju National University, 102 Jejudaehak-ro, Jeju-si, Jeju-do 63243, Republic of Korea. Tel: +82 647298556, Fax: +82 647563351, e-mail: sdjd1108@kaist.ac.kr and kimjh@jejunu.ac.kr
}

Key Words: MMP11, pancreatic cancer, prognosis, biomarker, whole blood.
(1). Although its prevalence is relatively low, it is the fourth leading cause of cancer-related death (2). In addition, the prognosis of pancreatic cancer patients remains unchanged in spite of significant improvements in overall survival rates for other cancers (3), which might be the reason why understanding pancreatic cancer becomes more significant.

One of the major features of pancreatic cancer is its high resistance to conventional cancer therapies including chemotherapy and radiation therapy. Another serious feature of pancreatic cancers is its early distant metastasis and locally abnormal progression that contribute to the relative rarity of surgery (4). A fundamental reason for the extremely poor prognosis may originate from the lack of an effective biomarker (5).

To date, human serum and plasma markers have been continuously developed due to their easier accessibility for sensible testing compared to tissue samples. In the last two decades, potential biomarkers examined for pancreatic cancer included CA19-9, DUPAN-2, CAM17.1, TPS, SPan-1, TAT1, POA, YKL-40, TUM2-PK, and matrix metalloproteinases (MMPs) (6). CA19-9 is recognized as the superior marker compared to others. However, sometimes it has poor sensitivity (41-86\%) and specificity (33-100\%) (7). Thus, the search for an effective biomarker continues for pancreatic cancer.

MMPs are a family of zinc-dependent endopeptidases that can lead to degradation of extracellular matrix (ECM) components (8). MMP11 is a member of the MMP family that plays a critical role in tumor angiogenesis, migration, survival, and degradation of connective tissues (9). MMP11 has been demonstrated as a critical factor in various malignant tumors such as lung cancer (10), head and neck cancer (11), gastric cancer (8), breast cancer (12), and pancreatic cancer (13) through clinicopathological approaches. In addition, MMP11 has been evaluated as a predictive serum based tumor marker in gastric cancer, breast cancer, colorectal cancer, and lung cancer (14). Despite continuous reports about cellular events of MMP11 
in various cancers, its underlying function as a soluble prognostic biomarker in pancreatic cancer remains unclear.

Considering latent actions of MMP11 in the tumor microenvironment, functional MMP11 should be examined as a possible prognostic biomarker for pancreatic cancer. In the present study, we provide the first evidence indicating that the levels of soluble MMP11 are higher in whole blood samples of pancreatic cancer patients compared to those of healthy donors. In addition, high levels of MMP11 were associated with poor prognosis in pancreatic cancer. Based on these results, we conclude that MMP11 is a promising prognostic biomarker for pancreatic cancer.

\section{Materials and Methods}

Selection of biomarker candidates. To select a candidate gene as pancreatic cancer biomarker, a total of 18,430 genes were sorted from nine data-sets of Gene Expression Omnibus (GEO) public microarray database as previously described (15). Selected genes that were significantly up-regulated in pancreatic cancer compared to those of normal pancreas were consecutively eliminated to identify a promising candidate as pancreatic cancer biomarker using The Human Protein Atlas database as described previously (16).

Gene expression analysis. Microarray expression profiles were obtained from Gene Expression Omnibus (GEO) and ArrayExpress (AE) public microarray database. Data-sets for pancreatic cancer included GSE19650, GSE39409, GSE42952, GSE16515, GSE17891, GSE32676, GSE22780, GSE15471, and E-MEXP-2780. Those for normal pancreas included GSE19650, GSE16515, EMEXP-1140, GSE32676, GSE22780, GSE15471, and GSE43346 in Oncopression database as described previously (17).

Cell culture and reagents. AsPC-1 (KCLB no. 21682), Capan-1 (KCLB no. 30079), Capan-2 (KCLB no. 30080), Miapaca-2 (KCLB no. 21420), Panc-1 (KCLB no. 21469), SNU-213 (KCLB no. 00213), and Detroit 551 (KCLB no. 10110) cells were obtained from the Korean Cell Line Bank (Seoul, Republic of Korea). CFPAC-1 (cat. no. CRL-1918) was purchased from American Type Culture Collection (ATCC, Manassas, VA, USA). The cells were grown in DMEM (cat. no. 12491-015, Gibco-BRL, Gaithersburg, MD, USA, for Panc-1, Miapaca-2, CFPAC-1, and Detroit 551) or RPMI 1640 (cat. no. 11875-093, Gibco-BRL Gaithersburg, MD, USA, for AsPC-1, Capan-1, Capan-2, and SNU-213) medium supplemented with $10 \%$ fetal bovine serum (cat. no. 12483-020, Gibco-BRL, Gaithersburg, MD, USA), $1 \times 10^{5}$ unit/l penicillin and $100 \mathrm{mg} / \mathrm{l}$ streptomycin (cat. no. 15140122, Gibco-BRL, Gaithersburg, MD, USA) at $37^{\circ} \mathrm{C}$ in a humidified atmosphere containing $5 \% \mathrm{CO}_{2}$ as described previously (18). H6c7 (cat. no. ECA001) was obtained from Kerafast (Boston, MA, USA) and grown as described previously (19). Under standard culture conditions, cells retained the typical morphologies of the original cell line throughout the time period required to perform the experiments reported in this study.

Tumor tissues array. To determine MMP11 expression levels in various normal and tumor tissues, human normal tissues blot I (cat. no. 1521, ProSci, Poway, CA, USA) and human tumor tissues blot
I (cat. no. 1542, ProSci, Poway, CA, USA) were used according to the manufacturer's instructions.

Western blot analysis. To evaluate protein levels of MMP11 in various human pancreatic cancer cells and whole blood samples of pancreatic cancer patients, western blot analysis was performed as described previously (20). Bands were quantified through densitometry analysis using ImageJ software.

Whole blood samples collection. A total of 12 different whole blood samples from pancreatic cancer patients and 16 different samples from healthy donors were purchased from Innovative (21) (Novi, MI, USA).

Enzyme-linked immunosorbent assay (ELISA). To measure soluble MMP11 in pancreatic cancer patients, whole blood samples were subjected to sandwich ELISA as described previously (22). Briefly, 96-well plates (cat. no. 32296, SPL, Daejeon, Republic of Korea) were coated with anti-MMP11 polyclonal antibody (cat. no. ab119284, Abcam, Cambridge, UK) at $1 \mu \mathrm{g} / \mathrm{ml}$. Bound soluble MMP11 was detected using anti-MMP11 monoclonal antibody (cat. no. sc517445, Santa Cruz Biotechnology, Santa Cruz, CA, USA). The absorbance was then measured at $450 \mathrm{~nm}$ using a microplate reader (Bio-Rad, Richmond, CA, USA).

Xenograft tumor model. BALB/c nude mice at 6-8 weeks of age were obtained from Orient (Seongnam, Republic of Korea). AsPC$1\left(1 \times 10^{7}\right)$ and Panc- 1 cells $\left(1 \times 10^{7}\right)$ were injected subcutaneously into the right flank as described previously (23). Once the tumor achieved a size of approximately $300 \mathrm{~mm} 3$, blood was drawn from xenograft models and non-tumor bearing mice. Body weight was recorded regularly every two days. Animal care and experiments were carried out in accordance with guidelines approved by Animal Bioethics Committee of Jeju National University (approval number: 2016-0049).

GSE data-set analysis. Gene expression data (E-MEXP-2780, GSE21501, GSE57495, GSE71729, GSE84219, PACA-AU_ PancreaticCancer-AU, GSE17891, GSE47368, GSE62452, GSE79668, PAAD-US_PancreaticCancer-TCGA_US and PAENAU_PancreaticCancerEndocrineneoplasms-AU) with prognosis information were downloaded from Gene Expression Omnibus, ArrayExpress, and ICGC data portal. Each probe was converted to EntrezID. Several probes for the same EntrezID were averaged. Quantile-quantile normalization was applied to all samples to remove batch effects. To test the prognostic value of a gene, samples were divided into two groups using median gene expression levels as threshold. Log-rank test was then performed using Graph Prism version 5. Several log-rank $p$-values from these data-sets were integrated into a single $p$-value using Liptak's weighted z-score method with square root of sample numbers as weight as described previously (24).

Statistical analyses. All data are presented as mean \pm standard deviation. Levels of significance for comparisons between two independent samples were determined by Student's $t$-test. Groups were compared by one-way analysis of variance (ANOVA) with Tukey's post-hoc test for significant main effects. SPSS $12.0 \mathrm{~K}$ for Windows (SPSS Inc., Chicago, IL, USA) was used for all statistical analyses. 


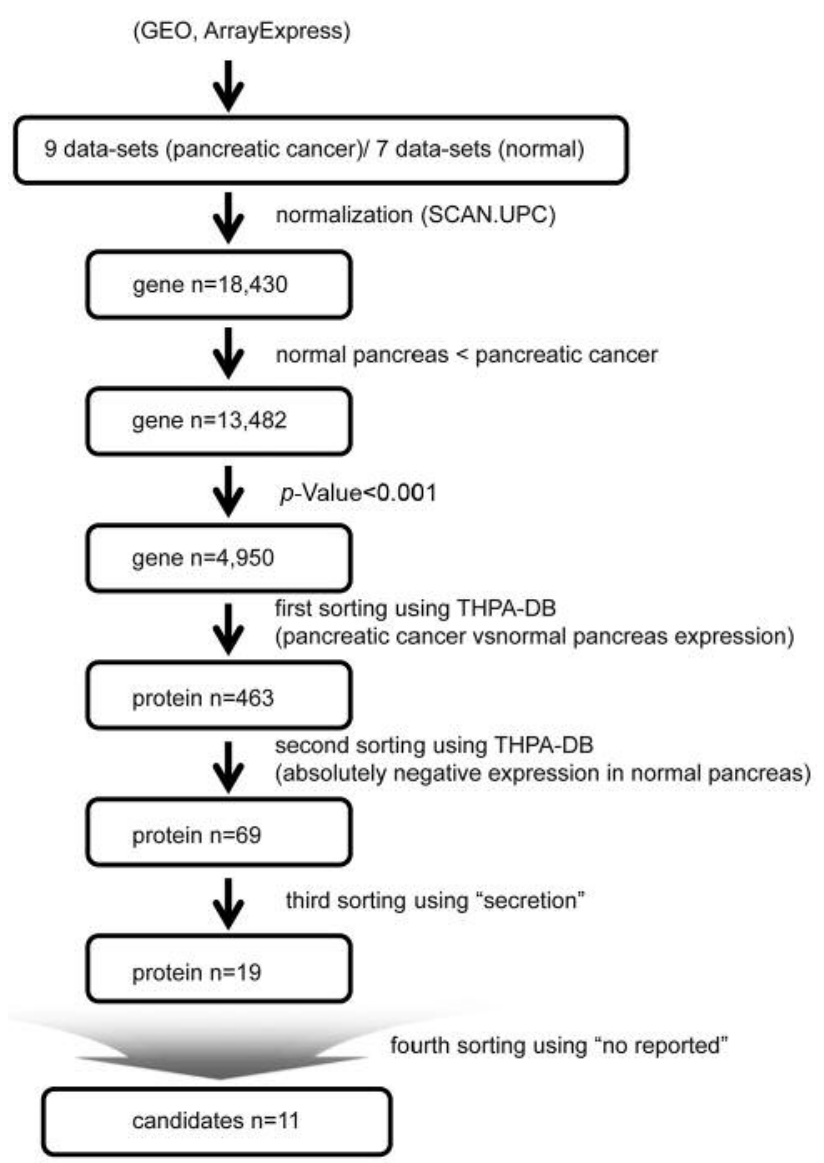

Figure 1. A schematic representation of candidate gene selection using consecutive eliminating method.

\section{Results}

Expression of MMP11 in human pancreatic cancer. To select a biomarker candidate for pancreatic cancer, a consecutive eliminating method was applied in a total of 18,430 genes from nine pancreatic cancer data-sets. Then, candidate genes were further sorted using The Human Protein Atlas database. A schematic representation of candidate genes selection is shown in Figure 1. A total of 11 biomarker candidates, including MMP11 that had the highest selection score were selected through the consecutive eliminating method.

Expression profiles of MMP11 in human pancreatic cancer and various normal tissues were obtained from the Oncopression database. Pancreatic cancer specifically expressed higher levels of MMP11 mRNA than various normal tissues, including pancreatic, gastric, renal, adrenal, oral, whole blood, colon, breast, ovarian, skin, lung, thyroid, head, muscle, liver, bone, lipid, and prostate samples (Figure 2A). To verify the specific expression of MMP11 in human pancreatic cancer, MMP11 protein expression levels were determined in various normal and tumor tissues by western blotting. MMP11 was specifically expressed in human pancreatic cancer, but not in various normal tissues (brain, colon, heart, kidney, liver, lung, pancreas, and spleen) or tumor tissues (brain, colon, kidney, liver, skin, spleen, stomach, and lung) (Figure 2B). In addition, MMP11 protein was highly expressed in seven human pancreatic cancer cells, but not in normal pancreas epithelial H6c7 cells and normal fibroblast Detroit-551 cells (Figure 2C). These results indicated that MMP11 expression is highly elevated in human pancreatic cancer.

Selection of soluble MMP11 in human pancreatic cancer. We next explored whether soluble MMP11 could be detected in whole blood samples. Xenograft models using AsPC-1 $(n=4)$ and Panc-1 $(n=4)$ cells were prepared and sandwich ELISA was performed to detect soluble MMP11 in whole blood samples from non-tumor bearing mice, AsPC-1 xenograft model, and Panc-1 xenograft model. Higher levels of soluble MMP11 were specifically detected in whole blood samples from AsPC-1 and Panc-1 xenograft models compared to those from non-tumor bearing mice $(n=4)$ (Figure 3A). Higher levels of soluble MMP11 could also be detected using sandwich ELISA in whole blood samples from pancreatic cancer patients $(n=12)$ compared to those from healthy donors $(n=16)$ (Figure 3B). Similar results were obtained by western blot analysis using whole bloods samples from pancreatic cancer patients and healthy donors (Figure 3C). These results suggested that soluble MMP11 could be detected in whole blood samples of pancreatic cancer patients using easy-to-do techniques.

MMP11 expression in human pancreatic cancer is associated with prognosis. To determine the involvement of MMP11 expression in the prognosis of pancreatic cancer patients, we analyzed GEO data-sets including overall survival days (OS). GSE57495 (low MMP11, MS=960.15 days; high MMP11, MS=533.30 days), PAAD-US-TCGA (low MMP11, $\mathrm{MS}=1332$ days; high MMP11, MS=568 days), GSE62452 (low MMP11, MS=1265.33 days; high MMP11, MS=416.7), and GSE78229 (low MMP11, MS=1832.6 days; high MMP11, MS=416.7 days) data-sets revealed that high expression of MMP11 was significantly associated with shorter median survival (MS) compared to low expression of MMP11 in patients with pancreatic cancer (Figure 4A-D). In addition, the prognostic relevance of MMP11 expression in pancreatic cancer patients was demonstrated using log-rank test and its non-parametric version (Figure 4E-H).

Previously, MMP11 has been proposed as a secondary prognostic biomarker following MMP7 in pancreatic cancer through Cox proportional hazard modeling (13). To determine the clinical value of MMP7 expression in 

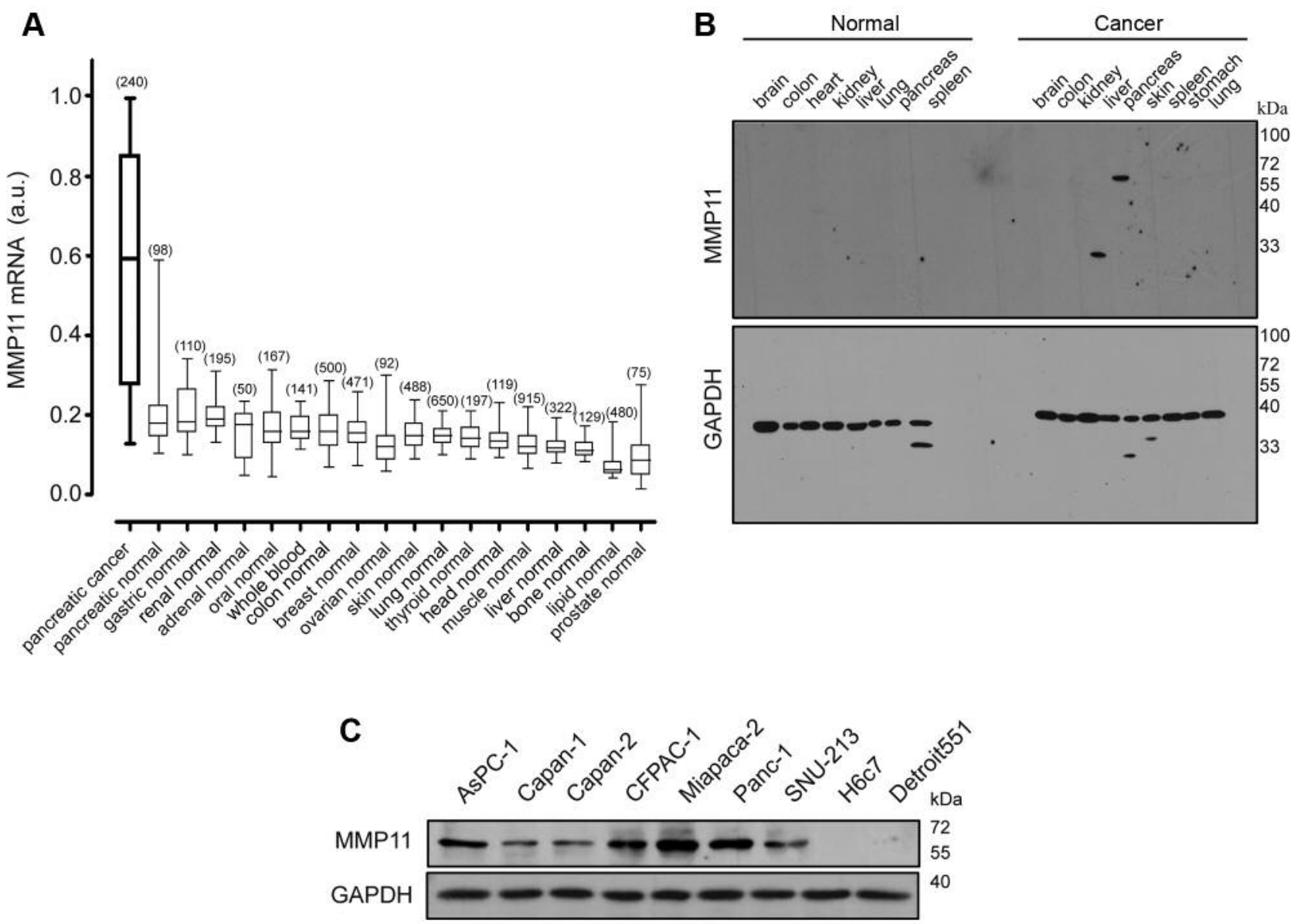

Figure 2. Expression of matrix metalloproteinase 11 (MMP11) in pancreatic cancer. A: mRNA levels of MMP11 in pancreatic cancer and various normal tissues were analyzed using the Gene Expression Omnibus (GEO), ArrayExpress (AE), and Oncopression databases (a.u. indicates arbitrary unit using the UPCs method, p-value was evaluated with Student's t test). B: Human Normal Tissue Blot I and Human Tumor Tissue Blot I were used to determine the expression levels of MMP11 in various normal and tumor tissues. GAPDH was measured as a control. Data is representative of two individual experiments. C: MMP11 expression in seven human pancreatic cancer cells, H6c7 cells, and Detroit551 cells was detected by western blot. GAPDH was measured as a control. Data is representative of three individual experiments.

pancreatic cancer, we analyzed GEO data-sets including overall survival days (OS). Only one data-set (GSE57495) revealed that high expression of MMP7 significantly shortened median survival (MS) compared to that of low expression of MMP7 in patients with pancreatic cancer (Figure 5). To further verify the clinical value of MMP11 expression in pancreatic cancer compared to that of MMP7, we analyzed Liptak's Z value from ten pancreatic cancer data-sets (PACA-AU, PAAD-US-TCGA-US, GSE84219, GSE79668, GSE71729, GSE62425, GSE57495, GSE215, GSE17891, and E-MEXP-2780). As shown in Table I, both MMP11 (Liptak's Z value: -1.7513) and MMP7 (Liptak's Z value: -1.1498 ) expression was associated with adverse prognosis of pancreatic cancer patients. However, expression of MMP11 ( $p=0.0399)$ was just statistically significant by Liptak's Z analysis, but not of MMP7 ( $p=0.1251)$.

Collectively, our results suggested that soluble MMP11 is highly expressed in human pancreatic cancer via adducing in vitro and in vivo evidence. In addition, clinical results revealed that high levels of MMP11 expression are associated with poor prognosis. Thus, MMP11 may be a promising prognostic biomarker for pancreatic cancer.

\section{Discussion}

Due to the deprivation of the opportunity for surgery and the lack of hope for improvement in prognosis (6), identification of effective biomarkers for pancreatic cancer is increasingly 
A

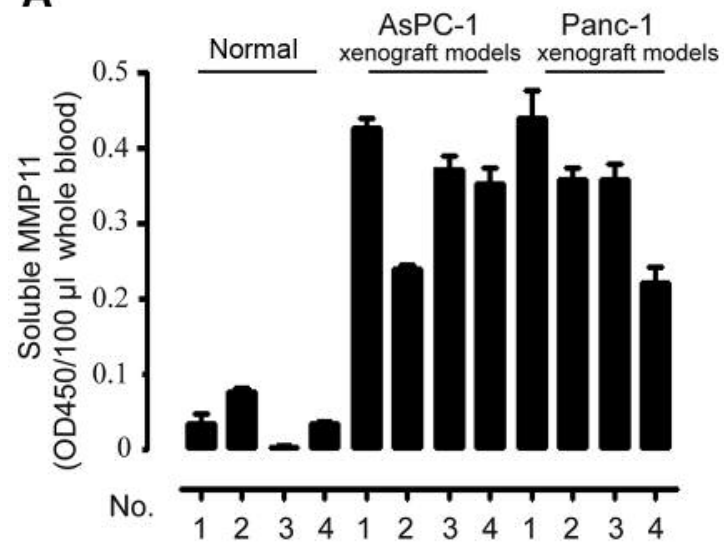

B

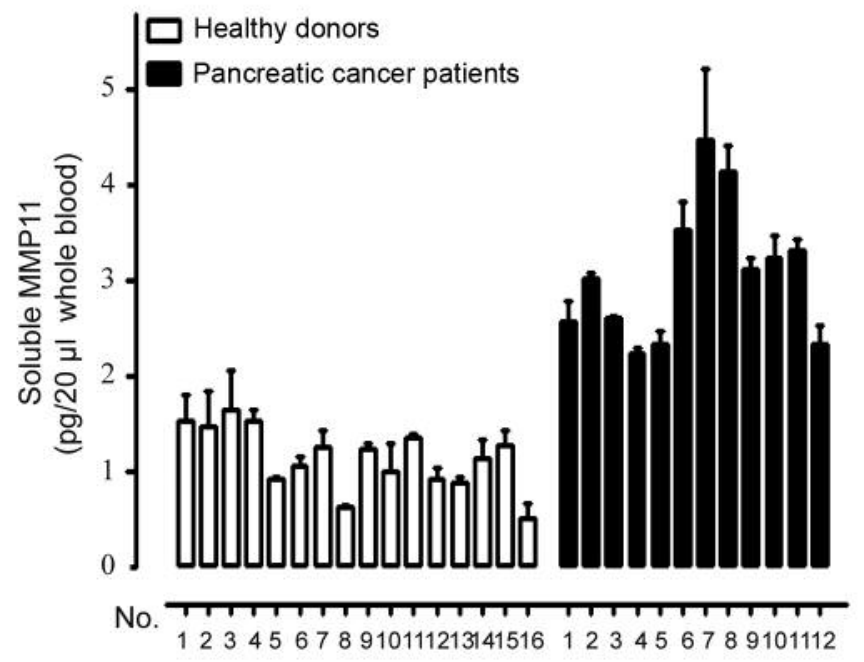

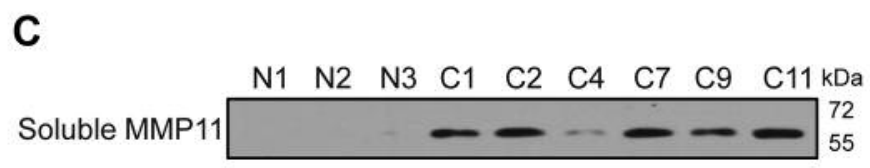

Figure 3. Detection of matrix metalloproteinase 11 (MMP11) using easily applicable techniques and whole blood samples of pancreatic cancer patients. A: Soluble MMP11 was detected by a sandwich ELISA in whole blood samples from Capan-1 and SNU-213 xenograft models (Normal means non-tumor bearing nude mice). B: Soluble MMP11 was detected by a sandwich ELISA in whole bloods samples from sixteen healthy donors (N1-N16) and twelve pancreatic cancer patients (C1-C12). C: Soluble MMP11 was detected by western blot analysis using whole bloods samples from healthy donors and pancreatic cancer patients. Data is representative of three individual experiments.

Table I. Prognosis associated with matrix metalloproteinase 11 (MMP11) and MMP7 expression in human pancreatic cancer using Liptak's Z analysis.

\begin{tabular}{|c|c|c|c|c|c|c|c|c|}
\hline \multirow[b]{2}{*}{ Data-set } & \multicolumn{4}{|c|}{ MMP11 } & \multicolumn{4}{|c|}{ MMP7 } \\
\hline & $\mathrm{Z}$ value & sqrt (n) & Low (n) & High (n) & $\mathrm{Z}$ value & sqrt (n) & Low (n) & $\operatorname{High}(\mathrm{n})$ \\
\hline PACA-AU & 0.993978 & 8.88819 & 50 & 29 & 0.336787 & 8.88819 & 52 & 27 \\
\hline PAAD-US-TCGA & 0.523939 & 11.7473 & 95 & 43 & 0.02528 & 11.7473 & 93 & 45 \\
\hline GSE84219 & 0.168449 & 5.47722 & 13 & 17 & -1.0022 & 5.47722 & 16 & 14 \\
\hline GSE79668 & -1.79555 & 7.141428 & 35 & 16 & 0.283781 & 7.141428 & 37 & 14 \\
\hline GSE71729 & 0.239568 & 11.090536 & 51 & 72 & 0.523776 & 11.090536 & 46 & 77 \\
\hline GSE62452 & 0.835607 & 8.062257 & 30 & 35 & 0.195503 & 8.062257 & 22 & 43 \\
\hline GSE57495 & -1.95008 & 7.93725 & 27 & 36 & -3.12724 & 7.93725 & 19 & 44 \\
\hline GSE21501 & 0.833271 & 10.0995 & 41 & 61 & 0.814344 & 10.0995 & 47 & 55 \\
\hline GSE17891 & 0.597931 & 5.196152 & 16 & 11 & 1.39348 & 5.196152 & 9 & 18 \\
\hline E-MEXP-2780 & 0.31002 & 5.47722 & 15 & 15 & 0.975664 & 5.47722 & 3 & 27 \\
\hline Liptak's Z value & \multicolumn{4}{|c|}{-1.751347} & \multicolumn{4}{|c|}{-1.149808} \\
\hline$p$-Value Liptak's z & \multicolumn{4}{|c|}{0.0399} & \multicolumn{4}{|c|}{0.1251} \\
\hline
\end{tabular}

sqrt (n) indicates square root values. 

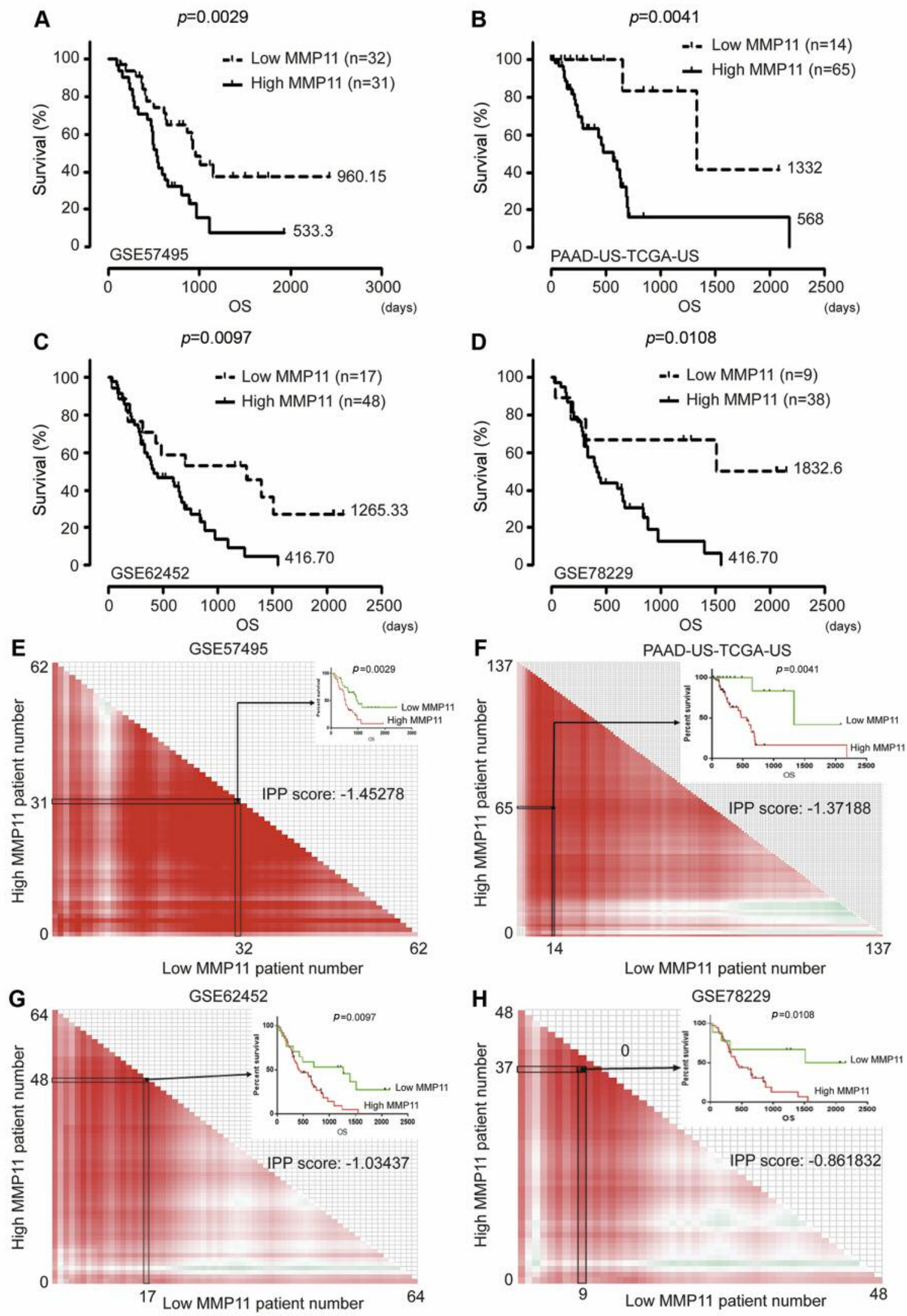
important. Although challenging therapies have been proposed by many researchers to inhibit pancreatic cancer, the overall survival of pancreatic cancer patients has not been improved in the last few decades (25). Such poor prognosis is associated with the absence of an effective biomarker $(3,26)$. In silico, in vitro and in vivo-clinical evidence obtained for the first time in the present study suggests that MMP11 is a promising prognostic biomarker in pancreatic cancer.

Due to remarkable development of bioinformatics related to cancers (27), we could access huge amount of information of pancreatic cancer. Eleven candidate biomarkers for pancreatic cancer were identified using the consecutive eliminating method with analysis of expression levels of genes or proteins in open-databases. In agreement with previous reports using open-databases to select biomarkers (28), our study also revealed that the consecutive eliminating method was distinctive based on three features comparing to other approaches using open databases. First, a total 18,430 genes from 9 data-sets for pancreatic cancer and 7 data-set for normal pancreas of GEO and AE public microarray database were adequately pooled due to the UPC normalization method. In the present study, selection of pancreatic cancer biomarkers was performed using the most abundant and up-to-date genes in open-data sources. Second, the consecutive eliminating method was orderly conducted in consideration of both mRNA and protein expression patterns. Various approaches for selecting cancer biomarkers have been performed reflecting specialization in one field, mRNA or protein expression (28). However, we nominated candidates for pancreatic cancer biomarkers that were significantly upregulated in pancreatic cancer compared to normal pancreas at the mRNA level. These were then consecutively eliminated based on protein levels using The Human Protein Atlas database. Thus, candidates for pancreatic cancer biomarkers were selected using organically combined sources of mRNA levels and protein levels. Lastly, 8 nominees [42\%, i.e. mesothelin; gene ID, 10232

$\leftarrow$

Figure 4. Prognosis associated with matrix metalloproteinase 11 (MMP11) expression in human pancreatic cancer. A-D: Overall survival of pancreatic cancer patients was analyzed using Kaplan-Meier curves depending on the differential expression of MMP11 in GSE57495, PAAD-US-TCGA-US, GSE62452 and GSE78229 data-sets ( $p$-value was calculated using Log-rank (Mantel-Cox) Test, Parentheses indicates median survival days). E-H: Prognostic relevance was analyzed using log-rank test and its non-parametric version of curves on the differential expression of MMP11 in GSE57495, PAAD-US-TCGA-US, GSE62452, and GSE78229 data-sets.
(29)] were coincided with previous reports, providing scientific confidence to the final 11 candidates.

Recently, expression levels of nine MMPs [1-3, 7-9, 11, 12, and 14] in pancreatic cancer have been reported using real-time RT-PCR and immunohistochemistry analysis. Immunohistochemistry has indicated that the expression levels of MMP7, -8, -9, and -11 were up-regulated. Using real-time RT-PCR, the mRNA levels of MMP11 in pancreatic cancer were found to be significantly increased compared to normal pancreas (13). Consistent with the previous report, the present study also revealed specific expression of MMP11 in human pancreatic cancers in silico and in vitro. As shown in Figure 2, higher levels of MMP11 mRNA were specifically detected in pancreatic cancer compared to various normal tissues. The levels of MMP11 protein were also specifically increased in pancreatic cancer compared to other cancers and normal tissues, implying that MMP11 may be a pancreatic cancer biomarker due to its unique expression patterns.

Currently, carbohydrate antigen 19-9 (CA19-9) is recognized as the most promising serum biomarker in pancreatic cancer. However, even CA19-9 is not a proper biomarker for pancreatic cancer due to its largely varied sensitivity and low specificity (30). The present study showed that MMP11 may be suitable as a biomarker for pancreatic cancer. Soluble MMP11 was verified as a potential soluble biomarker with significantly higher levels in whole blood samples of pancreatic cancer xenograft models $(n=8)$ and pancreatic cancer patients $(n=12)$ compared to non-tumor bearing models $(n=4)$ and healthy donors $(n=16)$. Although high serum levels of MMP11 have been reported in gastric cancer, breast cancer, colorectal cancer, and lung cancer (8), this is the first report showing that MMP11 might be a well-functional soluble biomarker using whole blood samples and easy-to-do techniques for pancreatic cancer patients. However, follow-up study with higher numbers of whole blood samples are required to conclusively determine the utility of MMP11 as a soluble biomarker in pancreatic cancer. To realize the "idea to clinic" of MMP11 as a substantial soluble biomarker for pancreatic cancer patients, the development of a user-friendly diagnostic kit needs to be established.

It has been previously reported that increased expression of MMP7, not MMP11, is a critical indicator of poor survival according to the Cox proportional hazards model (13). However, our results strongly suggested the clinical significance of MMP11 in pancreatic cancer. High expression of MMP11 significantly aggravated the prognosis of pancreatic cancer patients compared to low expression of MMP11 according to Kaplan-Meier curves for visual clarity and its non-parametric version of the curves using PAAD-USTCGA, GSE57495, GSE62452, and GSE78229 data-sets. However, high expression of MMP7 significantly aggravated 


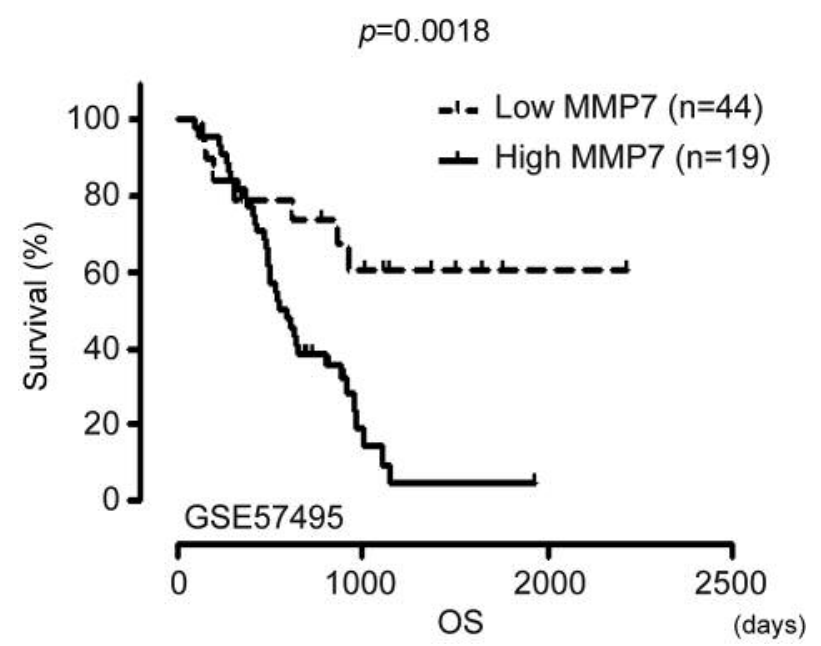

Figure 5. Prognosis associated with matrix metalloproteinase 7 (MMP7) expression in human pancreatic cancer. Overall survival of pancreatic cancer patients was analyzed using Kaplan-Meier curves depending on the differential expression of MMP7 in GSE57495 data-set (p-value was calculated using Log-rank (Mantel-Cox) Test.

the prognosis of pancreatic cancer patients compared to low expression of MMP7 only in the GSE57495 data-set, but not in PAAD-US-TCGA or GSE62452 datasets. Furthermore, increased MMP11 expression showed significantly higher association with clinical outcomes $(p<0.05)$ than MMP7 expression ( $p=0.125$ ) according to Liptak's Z analysis including sufficient numbers (low MMP11 expressed group, $\mathrm{n}=373$; high MMP11 expressed group, $\mathrm{n}=335$; low MMP7 expressed group, $n=344$; low MMP7 expressed group, $n=364$ ). To the best of our knowledge, this report clearly shows the impact of MMP11 expression in pancreatic cancer prognosis.

Collectively, our results provide the first evidence that soluble MMP11 could be specifically detected in whole blood samples of pancreatic cancer patients. In addition, its high expression showed significant association with poor prognosis in pancreatic cancer. Our results provide strong evidence that MMP11 may be a promising prognostic biomarker in pancreatic cancer.

\section{Conflicts of Interest}

There are no potential conflicts of interest regarding this study.

\section{Authors' Contributions}

JW. Lee planned the research, performed the experiments, and drafted the main manuscript. J. Lee arranged all data-sets. JW. Lee, J. Lee, and JH Kim analyzed and interpreted data. JW. Lee and J Kim supervised the project. All Authors read and approved the final manuscript.

\section{Acknowledgements}

This research was supported by Basic Science Research Program through the National Research Foundation of Korea (NRF) funded by the Ministry of Education (2017R1D1A1B03033899) and (2016R1A6A1A03012862).

\section{References}

1 Sohal DP, Walsh RM, Ramanathan RK and Khorana AA: Pancreatic adenocarcinoma: Treating a systemic disease with systemic therapy. J Natl Cancer Inst 106(3): dju011, 2014. PMID: 24563516. DOI: 10.1093/jnci/dju011

2 Siegel RL, Miller KD and Jemal A: Cancer statistics, 2016. CA Cancer J Clin 66(1): 7-30, 2016. PMID: 26742998. DOI: 10.3322/caac. 21332

3 Kleeff J, Korc M, Apte M, La Vecchia C, Johnson CD, Biankin AV, Neale RE, Tempero M, Tuveson DA, Hruban RH and Neoptolemos JP: Pancreatic cancer. Nat Rev Dis Primers 2: 16022, 2016. PMID: 27158978. DOI: 10.1038/nrdp.2016.22

4 Hutchinson L: Pancreatic cancer: Promise of doublet chemotherapy. Nat Rev Clin Oncol 13: 592, 2016. PMID: 27550853. DOI: 10.1038/nrclinonc.2016.138

5 Stathis A and Moore MJ: Advanced pancreatic carcinoma: Current treatment and future challenges. Nat Rev Clin Oncol 7(3): 163-172, 2010. PMID: 20101258. DOI: 10.1038/nrclinonc. 2009.236

6 Ray K: Pancreatic cancer: Biomarkers for the early detection of pdac. Nat Rev Gastroenterol Hepatol 14(9): 504-505, 2017. PMID: 28765582. DOI: 10.1038/nrgastro.2017.111

7 Zhang L, Sanagapalli S and Stoita A: Challenges in diagnosis of pancreatic cancer. World J Gastroenterol 24(19): 2047-2060, 2018. PMID: 29785074. DOI: 10.3748/wjg.v24.i19.2047

8 Yan D, Dai H and Liu JW: Serum levels of mmp-11 correlate with clinical outcome in chinese patients with advanced gastric adenocarcinoma. BMC Cancer 11: 151, 2011. PMID: 21513571. DOI: $10.1186 / 1471-2407-11-151$

9 Zhang X, Huang S, Guo J, Zhou L, You L, Zhang T and Zhao $\mathrm{Y}$ : Insights into the distinct roles of mmp-11 in tumor biology and future therapeutics (review). Int J Oncol 48(5): 1783-1793, 2016. PMID: 26892540. DOI: 10.3892/ijo.2016.3400

10 Yang H, Jiang P, Liu D, Wang HQ, Deng Q, Niu X, Lu L, Dai $\mathrm{H}$, Wang $\mathrm{H}$ and Yang $\mathrm{W}$ : Matrix metalloproteinase 11 is a potential therapeutic target in lung adenocarcinoma. Mol Ther Oncolytics 14: 82-93, 2019. PMID: 31024988. DOI: 10.1016/ j.omto.2019.03.012

11 Hsin $\mathrm{CH}$, Chou YE, Yang SF, Su SC, Chuang YT, Lin SH and Lin CW: Mmp-11 promoted the oral cancer migration and fak/src activation. Oncotarget 8(20): 32783-32793, 2017. PMID: 28427180. DOI: 10.18632 /oncotarget.15824

12 Gonzalez de Vega R, Clases D, Fernandez-Sanchez ML, Eiro N, Gonzalez LO, Vizoso FJ, Doble PA and Sanz-Medel A: Mmp11 as a biomarker for metastatic breast cancer by immunohistochemical-assisted imaging mass spectrometry. Anal Bioanal Chem 411(3): 639-646, 2019. PMID: 30218127. DOI: 10.1007/s00216-018-1365-3

13 Jones LE, Humphreys MJ, Campbell F, Neoptolemos JP and Boyd MT: Comprehensive analysis of matrix metalloproteinase and tissue inhibitor expression in pancreatic cancer: Increased 
expression of matrix metalloproteinase-7 predicts poor survival. Clin Cancer Res 10(8): 2832-2845, 2004. PMID: 15102692.

14 Yang YH, Deng H, Li WM, Zhang QY, Hu XT, Xiao B, Zhu HH, Geng PL and Lu YY: Identification of matrix metalloproteinase 11 as a predictive tumor marker in serum based on gene expression profiling. Clin Cancer Res 14(1): 74-81, 2008. PMID: 18172255. DOI: 10.1158/1078-0432.CCR-07-1179

15 Lee J, Hun Yun J, Lee J, Choi C and Hoon Kim J: Blockade of dual-specificity phosphatase 28 decreases chemo-resistance and migration in human pancreatic cancer cells. Sci Rep 5: 12296, 2015. PMID: 26212664. DOI: 10.1038/srep12296

16 Uhlen M, Fagerberg L, Hallstrom BM, Lindskog C, Oksvold P, Mardinoglu A, Sivertsson A, Kampf C, Sjostedt E, Asplund A, Olsson I, Edlund K, Lundberg E, Navani S, Szigyarto CA, Odeberg J, Djureinovic D, Takanen JO, Hober S, Alm T, Edqvist PH, Berling H, Tegel H, Mulder J, Rockberg J, Nilsson P, Schwenk JM, Hamsten M, von Feilitzen K, Forsberg M, Persson L, Johansson F, Zwahlen M, von Heijne G, Nielsen J and Ponten F: Proteomics. Tissue-based map of the human proteome. Science 347(6220): 1260419, 2015. PMID: 25613900. DOI: 10.1126/science. 1260419

17 Lee J and Choi C: Oncopression: Gene expression compendium for cancer with matched normal tissues. Bioinformatics 33(13): 20682070, 2017. PMID: 28881874. DOI: 10.1093/bioinformatics/btx121

18 Lee J, Han SI, Yun JH and Kim JH: Quercetin 3-o-glucoside suppresses epidermal growth factor-induced migration by inhibiting egfr signaling in pancreatic cancer cells. Tumour Biol 36(12): 93859393, 2015. PMID: 26109002. DOI: 10.1007/s13277-015-3682-x

19 Radulovich N, Qian JY and Tsao MS: Human pancreatic duct epithelial cell model for kras transformation. Methods Enzymol 439(1-13), 2008. PMID: 18374152. DOI: 10.1016/S00766879(07)0040-6

20 Lee J, Lee J, Yun JH, Jeong DG and Kim JH: Dusp28 links regulation of mucin $5 \mathrm{~b}$ and mucin 16 to migration and survival of aspc-1 human pancreatic cancer cells. Tumour Biol 37(9): 12193-12202, 2016. PMID: 27230679. DOI: 10.1007/s13277$016-5079-x$

21 Lee J, Lee J and Kim JH: Scattered dusp28 is a novel biomarker responsible for aggravating malignancy via the autocrine and paracrine signaling in metastatic pancreatic cancer. Cancer Letters 456: 1-12, 2019. DOI: 10.1016/j.canlet.2019.03.006
22 Lee J, Lee J, Yu H, Choi K and Choi C: Differential dependency of human cancer cells on vascular endothelial growth factormediated autocrine growth and survival. Cancer Lett 309(2): 145-150, 2011. DOI: 10.1016/j.canlet.2011.05.026

23 Lee J, Lee J, Yun JH, Choi C, Cho S, Kim SJ and Kim JH: Autocrine dusp28 signaling mediates pancreatic cancer malignancy via regulation of pdgf-a. Sci Rep 7(1): 12760, 2017. PMID: 28986588. DOI: 10.1038/s41598-017-13023-w

24 Zaykin DV: Optimally weighted z-test is a powerful method for combining probabilities in meta-analysis. J Evol Biol 24(8): 1836-1841, 2011. PMID: 21605215. DOI: 10.1111/j.14209101.2011.02297.x

25 Li C, Zhao Y, Yang D, Yu Y, Guo H, Zhao Z, Zhang B and Yin $\mathrm{X}$ : Inhibitory effects of kaempferol on the invasion of human breast carcinoma cells by downregulating the expression and activity of matrix metalloproteinase-9. Biochem Cell Biol 93(1): 16-27, 2015. PMID: 25453494. DOI: 10.1139/bcb-2014-0067

26 Sidaway P: Pancreatic cancer: New biomarkers improve standard screening. Nat Rev Clin Oncol 14(5): 262, 2017. PMID: 28290493. DOI: 10.1038/nrclinonc.2017.35

27 Casci T: Bioinformatics: Next-generation omics. Nat Rev Genet 13(6): 378, 2012. PMID: 22549153. DOI: $10.1038 / n r g 3250$

28 Zhang X, Sun XF, Cao Y, Ye B, Peng Q, Liu X, Shen B and Zhang H: CBD: A biomarker database for colorectal cancer. Database (Oxford) 2018: bay046, 2018. PMID: 29846545. DOI: 10.1093/database/bay046

29 Kendrick ZW, Firpo MA, Repko RC, Scaife CL, Adler DG, Boucher KM and Mulvihill SJ: Serum igfbp2 and msln as diagnostic and prognostic biomarkers for pancreatic cancer. HPB (Oxford) 16(7): 670-676, 2014. PMID: 24308545. DOI: 10.1111/hpb.12199

30 Swords DS, Firpo MA, Scaife CL and Mulvihill SJ: Biomarkers in pancreatic adenocarcinoma: Current perspectives. Onco Targets Ther 9: 7459-7467, 2016. PMID: 28003762. DOI: 10.2147/OTT.S100510
Received September 24, 2019

Revised October 2, 2019

Accepted October 7, 2019 